\title{
Natural history of cervical intraepithelial neoplasia in pregnancy: postpartum histo-pathologic outcome and review of the literature
}

\author{
Mariella Mailath-Pokorny ${ }^{1}$, Richard Schwameis², Christoph Grimm², Alexander Reinthaller ${ }^{2,3}$
} and Stephan Polterauer ${ }^{2,3^{*}}$

\begin{abstract}
Background: To study the natural history of cervical intraepithelial neoplasia (CIN) during pregnancy and to compare the rates of persistence, progression and regression of CIN by colposcopically guided biopsy (CGB) during pregnancy with outcome in non-pregnant-women.

Methods: A retrospective analysis of all pregnant women diagnosed with CIN at our outpatient clinic between 2005 and 2010 was performed. A CGB for histo-pathological analysis was obtained in all participants and observational management was performed. The histo-pathologic findings of initial and postpartum visits were collected. Rates of persistence, progression and regression of CIN were assessed. Results were compared to a matched control group of non-pregnant women where observational management was performed for at least three months. In addition a review of the literature and pooled analysis of published data was performed.
\end{abstract}

Results: A total of 51 pregnant women with CIN were included into analysis. CIN 1, 2, and 3 was diagnosed by CGB in 33.3, 13.7 and $52.9 \%$ of all pregnant women, respectively. The postpartum histo-pathologic evaluation of the pregnant cohort revealed a significantly higher tendency to spontaneous regression ( 56.9 versus $31.4 \%, p=0.010$ ) and a considerably, but not significantly higher complete remission rate $(41.2$ versus $27.5 \%, p=0.144)$ when compared to the non-pregnant cohort. In addition, we observed a significantly lower CIN persistence rate than in the non-pregnant cohort (39.2 versus $58.8 \%, p=0.048$ ). The progression rate was notably low in the pregnant cohort (3.9\%) and no progression to invasive cancer was observed.

Conclusions: CIN lesions show considerably high spontaneous regression rates postpartum. Once presence of invasive cancer is ruled out definitive treatment can be deferred to the postpartum period.

Keywords: Cervical intraepithelial lesion, Pregnancy, Regression, Persistence, Regression, HPV

\footnotetext{
* Correspondence: stephan.polterauer@meduniwien.ac.at

${ }^{2}$ Gynecologic Cancer Unit, Comprehensive Cancer Center, Department of

Obstetrics and Gynecology, Division of General Gynecology and Gynecologic

Oncology, Medical University of Vienna, Vienna, Austria

${ }^{3}$ Karl Landsteiner Institute for General Gynecology and Experimental

Gynecologic Oncology, Vienna, Austria

Full list of author information is available at the end of the article
} 


\section{Background}

The prevalence of cervical intraepithelial neoplasia (CIN) in the population of pregnant women is approximately $1 \%$ [1]. The recent literature recommends a cervical cytology-based screening as part of routine prenatal care, since most of the precursor lesions of cervical cancer occur in young women within the child bearing age [2]. Moreover, the incidence of persistent HPV infection, which is associated with the development of CIN is highest in women aged 20-25 years [3-6]. The trend of managing intraepithelial abnormalities during pregnancy has changed over the years from an aggressive surgical management to a more conservative approach. Once invasive disease has been ruled out, observational management with regular gynecological examinations including PAP-smear, colposcopy and colposcopy-guided biopsy (CGB) has been proven to be safe and is recommended by expert groups [7-10]. However the published literature reveals heterogeneous data about regression rates in pregnant women with CIN I between 32 and $69 \%$ and CIN II-III between 16.7 to almost $70 \%$, respectively $[7,9,11-14]$. Additionally persistence rates, ranging from 38.4 up to $70 \%[8,16,17]$ have been reported, thus leading to the recommendation of a close postpartum follow-up [8, 11, 16, 18].

The aim of the present report was to study the natural history of histo-pathologically confirmed CIN lesions in pregnant women and to assess postpartum rates of regression, persistence, and progression. We used a robust data set obtained at a large, single center with a close follow-up of patients. In addition, a review of the current literature and pooled data analysis was performed.

\section{Methods}

All consecutive patients, that were initially referred to the institution's outpatient clinic due to an abnormal PAP test result and had a diagnostic work up including cervical punch biopsy, were documented. Data of all women, who were diagnosed with CIN between January 1, 2005 and December 31, 2010, were collected and analyzed. All pregnant women were identified and included into this study. A control group of non-pregnant women matched for CIN grade was selected. Women of both groups had observational management for at least 3 months and were diagnosed within the same observation period. The institution's electronic documentation system was used for data collection and patients' charts were analyzed to assess demographic and clinicalpathological data. In the course of routine diagnostic work-up at the patients' first visit at the institution a questionnaire was used to inquire patients' medical history and demographic data. Initial diagnostic work-up for patients with abnormal PAP test results included the following: colposcopy, repeated PAP smears, and CGB of all suspicious lesions. Women with pathological cytology and negative or equivocal colposcopy were biopsied from all four quadrants of the uterine cervix. Human papilloma virus (HPV) testing using the Hybrid Capture ${ }^{\odot}$ HPV DNA test was performed based on the physician's decision. In pregnant women clinical examinations including PAP smear and colposcopy with or without CGB were performed in each pregnancy trimester in order to rule out presence of invasive disease. Postpartum visits were scheduled approximately eight weeks after delivery and CIN status was assessed using CGB. Non-pregnant women with CIN were seen every 3-6 months and examined with colposcopy and CGB. We assessed CIN persistence, regression, and progression rates within the observational time period. Results of cervical punch biopsies were used for the assessment of natural history of CIN and CIN status in pregnant and non-pregnant women. Persistence, regression, and progression rates at last follow-up visit were assessed based on histologic findings from biopsy or conization specimens. Regression at a subsequent visit was defined as a lower grade lesion than that found at the initial visit. Persistent disease was defined as CIN of same grade as found at the initial diagnostic work up. Disease progression was defined as histologic evidence of higher CIN grade at subsequent visit compared to the initial visit. HPV clearance was assessed, if HPV status was available at the beginning and the end of the observation period.

We used descriptive statistics for visualization of patients' demographic data. Values are given as mean (standard deviation [SD]) when normally distributed or as median (range) at presence of skewed distribution. $P$-values of $<0.05$ were considered statistically significant. We used the statistical software SPSS 21.0 for Windows (SPSS 21.0, SPSS Inc, Chicago, IL, USA) for statistical analyses. The impact of pregnancy on qualitative outcome variables (i.e., regression vs. no regression, complete regression vs. no complete regression, persistence vs. no persistence and progression vs. no progression) was assessed by using the Chi-squared test. Binary logistic regression analysis was performed to assess odds ratios and $95 \%$ confidence intervals (95\% CI) as well as to adjust for covariables like histological grade, age, and smoking status (Table 2). The impact of histological grade of pregnant women at time of diagnosis on qualitative outcome variables (i.e., regression vs. no regression, complete regression vs. no complete regression, persistence vs. no persistence and progression vs. no progression) was assessed. Binary logistic regression analysis was performed to assess odds ratios and $95 \%$ confidence intervals (95 \% CI) (Table 3). In January 2014, a Pubmed search using the terms "cervical intraepithelial neoplasia" or "CIN" or "cervical dysplasia" and "pregnancy" or "pregnant women" was performed to identify articles on this 
topic published in the English literature. Only studies reporting histo-pathological postpartum outcome were selected and reviewed. A pooled analysis of regression, persistence, and progression rates were performed. For Table 4 data from published articles were assessed and combined in the pooled analysis, a formal meta-analysis was not performed.

Study approval was obtained from the institutional review board (IRB) of the Medical University of Vienna (ECS 1213-2011). Due to the retrospective design of the present study the institution's IRB granted a waiver of consent. Therefore no informed consent was obtained.

\section{Results}

Within the study period 51 pregnant women with histologically proven CIN were identified and included into the analysis. We observed CIN I in 17 (33.3 \%), CIN II in 7 (13.7 \%) and CIN III in 27 (52.9 \%) pregnant women, respectively. Mean (standard deviation (SD)) gestational age at diagnosis was 15.0 (6.3) weeks. During the same period of diagnosis we identified a control group of 51 consecutive non-pregnant women, which was matched by CIN grade at diagnosis in order to compare the natural history of $\mathrm{CIN}$ in pregnant versus non-pregnant women. Characteristics of pregnant and non-pregnant women with CIN are shown in Table 1. Parity was significantly higher in the group of pregnant patients. No statistically significant difference was found between the cohorts with respect to HPV status, age and smoking history. Mean (SD) follow-up time in pregnant women and non-pregnant women was 36.4 (29.0) and 51.6 (30.5) weeks, respectively. The main difference in

Table 1 Patients' characteristics at study inclusion

\begin{tabular}{llll}
\hline Parameter & $\begin{array}{l}\text { Pregnant } \\
\text { Women } \\
\text { N (\%), mean (SD) }\end{array}$ & $\begin{array}{l}\text { Non-pregnant } \\
\text { Women } \\
\mathrm{N}(\%), \text { mean (SD) }\end{array}$ & \\
\hline Total & 51 & 51 & (malue \\
Histological grade & & $17(33.0)$ & \\
CIN 1 & $17(33.0)$ & $7(13.7)$ & \\
CIN 2 & $7(13.7)$ & $27(52.9)$ & \\
CIN 3 & $27(52.9)$ & & \\
HPV high-risk & & $38(92.7)$ & \\
Positive & $25(86.2)$ & $3(7.3)$ & \\
Negative & $4(13.8)$ & 10 & 0.675 \\
Missing & 21 & $30.6(6.0)$ & 0.005 \\
Age & $30.2(5.5)$ & $1.03(1.3)$ & 0.221 \\
Parity & $1.88(1.2)$ & & \\
Smoking Status & & $14(60.9)$ & \\
Smoker & $21(70.0)$ & $9(39.1)$ & \\
Non-smoker & $9(30.0)$ & & \\
\hline
\end{tabular}

observation time was observed in pregnant and nonpregnant women with CIN 3 ( $p=0.003)$. Mean (SD) observation time was 20.4 (19.5) weeks and 47.5 (30.1) weeks in non-pregnant and pregnant women with CIN 3 , respectively $(p<0.001)$. In Table 2 the rates of persistence, progression, regression, and complete remission of CIN diagnosed by CGB at follow-up examination for pregnant and non-pregnant women are provided. Adjusted and unadjusted odds ratios and $95 \%$ confidence intervals are shown. Pregnant women showed significantly higher spontaneous regression rates. For pregnant and non-pregnant women HPV clearance rates $(95 \%$ CI) were $26.7 \%(0.72-2.10)$ and $38.9 \%(0.36-1.52)$, respectively. In addition we observed a significantly lower persistence rate than in non-pregnant controls. The progression rate was notably low in the pregnant cohort and no cases of invasive cancer were observed within the observation period. All of the 51 pregnant women had regular postpartum follow-up visit starting 8 weeks after delivery with a mean (SD) follow up time of 43.9 (30.6) weeks after delivery. In our series we observed 50 live births and one pregnancy loss. A cesarean section rate of $34.1 \%$ was noted. Rates of histo-pathological outcome parameters of pregnant patients broken down by CIN grade within the observation period are shown in Table 3. In total, 24 (47.1\%) patients of the pregnant cohort underwent conisation at a mean (SD) time of 44 (22.8) weeks postpartum. Table 4 provides a review of the published literature and pooled analysis of studies reporting outcome rates of CIN in pregnancy where histo-pathological outcome data were available. In total 364 articles were found matching the search criteria. Only data from studies where histological baseline and outcome information was available were summarized together with the presents study within the pooled analysis $(n=8)$. Pooled analysis revealed overall regression, persistence, and progression rates of 46.8, 43.6, and $9.6 \%$ for pregnant women with CIN, respectively [7-9, $11,15-17,19]$.

\section{Discussion}

The present study revealed higher spontaneous regression rates and lower persistence rates for CIN I-III in pregnant women when compared to non-pregnant women. We observed a notably low rate of disease progression and no cases of progression to invasive disease during the postpartum follow-up period. Our data support the opinion that once invasive disease can be excluded by colposcopy and CGB, definitive CIN therapy in pregnant women can be deferred until after delivery. In the current guideline of the American Society of Colposcopy and Cervical Pathology (ASCCP) observational management for women diagnosed with $\mathrm{CIN}$ in pregnancy is recommended [10]. 
Table 2 Rates of histo-pathological outcome parameters within the observation period

\begin{tabular}{|c|c|c|c|c|c|}
\hline \multirow[t]{2}{*}{ Parameter } & \multirow{2}{*}{$\begin{array}{l}\text { Pregnant Women } \\
N(\%)\end{array}$} & \multirow{2}{*}{$\begin{array}{l}\text { Non-pregnant Women } \\
\text { N (\%) }\end{array}$} & \multirow[t]{2}{*}{$p$-Value } & \multirow[t]{2}{*}{ OR $(95 \% \mathrm{Cl})$} & \multirow[t]{2}{*}{ AOR $(95 \% C l)^{a}$} \\
\hline & & & & & \\
\hline Total & 51 & 51 & & & \\
\hline Persistence & $20(39.2)$ & $30(58.8)$ & 0.048 & $0.45(0.20-0.99)$ & $0.21(0.05-1.02)$ \\
\hline Progression & $2(3.9)$ & $5(9.8)$ & 0.240 & $0.38(0.07-2.03)$ & $0.28(0.02-4.45)$ \\
\hline Regression & $29(56.9)$ & $16(31.4)$ & 0.010 & $2.88(1.28-6.49)$ & $7.02(1.33-37.22)$ \\
\hline Complete Remission & $21(41.2)$ & $14(27.5)$ & 0.144 & $1.85(0.81-4.24)$ & $4.12(0.54-31.43)$ \\
\hline
\end{tabular}

$O R$ odds ratio, $A O R$ adjusted odds ratio, $95 \% \mathrm{Cl} 95 \%$ confidence interval

a adjusted for covariables: histological grade, age, and smoking status

The incidences of CIN and HPV infection in pregnant women are comparable to that of non-pregnant women $[20,21]$. The initial obstetrical consultation provides an excellent opportunity to detect patients with abnormal PAP smears [2, 20, 21]. Historically, women with highgrade CIN were treated by cone biopsy during pregnancy $[22,23,24]$. Several studies reported that cone biopsy in pregnancy is associated with an impaired pregnancy outcome $[25,26]$. Other reports showed that loop electrosurgical excision procedures are safe during pregnancy with a miscarriage rate $<1 \%$ [17]. Due to the low rates of progression during pregnancy it is nowadays accepted that most patients may safely undergo expectant management if invasive disease has been ruled out [13, 27]. Within the last decades, several authors studied the natural history of CIN diagnosed during pregnancy with varying outcome (Table 4).

Our study reports a significantly higher postpartum regression rate of CIN in the group of pregnant patients than in the non-pregnant group. The high regression rate is in accordance to recent previous studies, which report regression rates between 37 and $74 \%$ for pregnant women at the time of post-partum follow-up [7-9, $12,14,15,19]$. Additionally we observed a $82 \%$ regression rate of CIN I lesions, which is even higher than the results reported by Serati et al. [15]. Nevertheless, as reflected by the $95 \% \mathrm{CI}$ the results need to be interpreted with caution due to the limited number of cases. Many theories to explain these high regression rates can be found in the recent literature and among them most commonly the ones discussed below. Some authors hypothesized that, in women with HPV infection, the typical hormonal pattern during pregnancy induces a viral activation that subsequently leads to increased spontaneous regression rates after delivery [15]. Other authors speculated that regression rates could theoretically be increased by the performance of multiple cervical biopsies in the antepartum evaluation, thereby giving the appearance of spontaneous regression [14]. On the other hand in the study by Ahdoot et al. the rate of regression was higher for women who had no histologic cervical biopsy as part of the antenatal evaluation compared with women who underwent a biopsy (70 versus $52 \%$ ) [14]. Alternatively other studies suggested a correlation between CIN course and mode of delivery and found a higher rate of regression of cervical dysplasia in association with vaginal delivery compared with cesarean section $(67$ versus $13 \%)$ [14, 28]. A possible mechanism for this finding may be the loss of the dysplastic cervical epithelium during cervical ripening and the passage of the fetus through the birth canal $[9,29]$. Other studies have not reported any differences in regression rates among patients who delivered vaginally or by cesarean section $[7,30]$. In the present study, no differences between regression rates after vaginal delivery (53.6\%) and after cesarean section (66.6 \%) were observed. However, due to the limited number of pregnant women when broken-down by mode of delivery we were not able to investigate the association between regressions rates and mode of delivery adequately.

In pregnant women with a diagnosis of CIN II-III the progression rate to invasive carcinoma ranges from 2 to

Table 3 Rates of histo-pathological outcome parameters of pregnant patients broken down by CIN grade

\begin{tabular}{llllll}
\hline Parameter & CIN 1 & CIN 2 & CIN 3 & N-Value & OR (95 \% Cl) \\
\hline Total & N (\%) & N (\%) & 7 & 27 & \\
$\quad$ Persistence & 17 & $1(14.3)$ & $17(63.0)$ & 0.002 & $4.01(1.68-9.61)$ \\
Progression & $2(11.8)$ & $1(14.3)$ & 0 & 0.32 & $4.02(0.07-2.38)$ \\
Regression & $1(5.9)$ & $5(71.4)$ & $10(37.0)$ & 0.005 & $0.34(0.16-0.72)$ \\
Complete Remission & $14(82.4)$ & $5(71.4)$ & $2(7.4)$ & 0.001 & $0.13(0.05-0.33)$ \\
\hline
\end{tabular}


Table 4 Review of the literature and pooled analysis of studies with reported histo-pathological outcome

\begin{tabular}{|c|c|c|c|c|c|c|}
\hline Author, Date & $N$ & Population & Analysis & Regression & Persistence & Progression \\
\hline Lurain [19] & 53 & Pregnant women with CIN I-III & Retrospective & $77.4 \%$ & $22.6 \%$ & $0 \%$ \\
\hline Yost [7] & 153 & Pregnant women with CIN II-III & Retrospective & $69.3 \%$ & $26.8 \%$ & $3.9 \%$ \\
\hline Palle [16] & 142 & Pregnant women with CIN I-III & Retrospective & $25 \%$ & $47 \%$ & $28 \%$ \\
\hline Vlahos [8] & 78 & Pregnant women with CIN II-III & Retrospective & $61.6 \%$ & $38.4 \%$ & $0 \%$ \\
\hline Paraskevaides [9] & 64 & Pregnant women with CIN II-III & Retrospective & $37.5 \%$ & $59.4 \%$ & $3.1 \%$ \\
\hline Serati [15] & 36 & Pregnant women with CIN II-III & Prospective & $47.3 \%$ & $52.7 \%$ & $0 \%$ \\
\hline Coppolillo [11] & 30 & Pregnant women with CIN II-III & Retrospective & $16.7 \%$ & $70.0 \%$ & $13.3 \%$ \\
\hline Kärrberg [17] & 163 & Pregnant women with CIN I-III & Prospective & $33.1 \%$ & $54.6 \%$ & $12.3 \%$ \\
\hline This study & 51 & Pregnant women with CIN I-III & Retrospective & $56.9 \%$ & $39.2 \%$ & $3.9 \%$ \\
\hline Pooled analysis & 770 & Pregnant women with CIN I-III & Pooled & $46.8 \%$ & $43.6 \%$ & $9.6 \%$ \\
\hline
\end{tabular}

$28 \%$, according to different reports. Ackermann et al. and Everson et al. have reported progression rates of $2.4 \%$ [31] and $3 \%$ [32], whereas Coppola et al. [30], Kaplan et al. [33], Kaerrberg et al. [17] and Coppolillo et al. [11] reported higher rates of $8,11,12$ and $13 \%$ respectively. Palle et al. reported the highest rate of progression to invasive carcinoma during the postpartum period with an incidence of $28 \%$ [16]. In our study, the progression rate was notably low in the pregnant cohort. We observed an incidence of 5.9 and $14.3 \%$ progression in the CIN I and CIN II group, respectively. No case of invasive carcinoma was diagnosed postpartum, which is consistent with the findings of other authors $[7,9,15]$. In addition we observed a significantly lower persistence rate than in non-pregnant controls (39.2 versus $58.8 \%$ ). Our persistence rate is also lower than that reported previously ranging from 47 to $89 \%$ [11, 16, 30, 33]. This difference might be related to variations in the methods used as well as the follow-up time of the study cohort. Additionally one may argue that these inconsistent data might be related to disparities in HPV prevalence and HPV types in the different study populations. Unfortunately, due to the retrospective study design, we cannot present typespecific HPV data. Additionally a notable number of patients did not have any HPV status assessment. Previous studies have shown that CIN is more common among smokers [34]. In our study we found a notable high smoking rate of $70 \%$ in the pregnant population, which was slightly higher compared to the $61 \%$ smoking rate in the non-pregnant cohort. This high rate does not clearly support the theory that regression rates are lower among pregnant smokers [12], although our dataset is clearly too small to draw any conclusion. The high rate of smokers within our cohort has to be kept in mind when interpreting our findings with respect to other populations.

Limitations of our study include the retrospective study design as well as the limited number of cases. In addition the limited data on HPV status and the lack of typespecific HPV data has to be recognized as an important limitation of the present study. We did not consider cytology results in the present study. Both HPV status and cytology results might give important additional clinical information and might influence management decisions. Another limitation might be caused by potential surveillance bias. Pregnant women are potentially more likely to participate in cytology screening programs than non-pregnant women. One might argue that therefore the pregnant women group might contain more early lesions with potentially benign biologically behavior. On the other hand, a recent study compared screening results in pregnant and non-pregnant women and could not detect any difference between CIN incidence [35]. Follow-up algorithms slightly differed between pregnant and non-pregnant women. In addition follow-up times were significantly longer for pregnant women. Especially non-pregnant women with CIN3 had a significantly shorter observation period than pregnant women. These findings might have positively influenced the rate of regression in the pregnant cohort and has to be regarded as relevant limitation of our study. However, we present data from a single, tertiary referral center using a robust, prospectively obtained database with a complete follow-up of the study patients. Additionally the complete cytologic and histo-pathologic work-up was performed by one laboratory, which may rule out interobserver variablity and therefore may reflect a strength of our study. We think that our findings are clinically meaningful and can be interpreted in the light of other studies reviewed in this report.

\section{Conclusion}

Our study suggests that a conservative management of $\mathrm{CIN}$ in pregnancy is safe since we report high regression rates and low progression rates after delivery. Our findings support the recommendation of the ASCCP that conservative management of CIN in pregnancy is safe. Within the postpartum period relatively high regression rates of CIN and notably low rates of disease progression were observed in our cohort. 


\section{Competing interests}

We state that there are no financial or other relationships that might lead to a conflict of interest.

\section{Authors' contributions}

MMP and SP participated in the design of the study, performed the statistical analysis and contributed to analysis and interpretation of data. AR and CG were involved in drafting the manuscript and revising it critically for important intellectual content. RS was involved in acquisition of data and data quality assessment. All authors read and approved the final manuscript.

\section{Acknowledgments}

The authors want to acknowledge the support of Luisa Pospischil, Anna-Maria Parger, Marie-Kristin Nozicka, Marina Gärner, and Hülya Gülmez in collecting clinical data. We thank Christian Göbl, MD PhD MSc (Medical Biometry), Department of Obstetrics and Gynecology, Division of Obstetrics and feto-maternal Medicine for helpful discussions and statistical advices. Publication fee was provided by the Karl Landsteiner Institute for General Gynecology and Experimental Gynecologic Oncology, Austria.

\section{Funding}

Publication costs were covered by the Karl Landsteiner Institute for General Gynecology and Experimental Gynecologic Oncology, Austria.

\section{Author details}

'Department of Obstetrics and Gynecology, Division of Obstetrics and Feto-maternal Medicine, Medical University of Vienna, Vienna, Austria. ${ }^{2}$ Gynecologic Cancer Unit, Comprehensive Cancer Center, Department of Obstetrics and Gynecology, Division of General Gynecology and Gynecologic Oncology, Medical University of Vienna, Vienna, Austria. ${ }^{3}$ Karl Landsteiner Institute for General Gynecology and Experimental Gynecologic Oncology, Vienna, Austria.

\section{Received: 17 March 2015 Accepted: 4 April 2016 Published online: 07 April 2016}

\section{References}

1. Insinga RP, Glass AG, Rush BB. Diagnoses and outcomes in cervical cancer screening: a population-based study. Am J Obstet Gynecol. 2004;191:105-13.

2. Frega A, Scirpa P, Corosu R, Verrico M, Scarciglia ML, Primieri MR, et al. Clinical management and follow-up of squamous intraepithelial cervical lesions during pregnancy and postpartum. Anticancer Res. 2007;27:2743-6.

3. Bano F, Kolhe S, Zamblera D, Jolaoso A, Folayan O, Page L, et al. Cervical screening in under 25s: a high-risk young population. Eur J Obstet Gynecol Reprod Biol. 2008:139:86-9.

4. Bosch FX, de Sanjosé S. The epidemiology of human papillomavirusinfection and cervical cancer. Dis Markers. 2007:23:213-27.

5. Lowy DR, Schiller JT. Prophylactic human papillomavirus vaccines. J Clin Invest. 2006:116:1167-73.

6. Snijders PJ, Steenbergen RD, Heideman DA, Meijer CJ. HPV-mediated cervical carcinogenesis: concepts and clinical implications. J Pathol. 2006; 208:152-64.

7. Yost NP, Santoso JT, McIntire DD, Iliya FA. Postpartum regression rates of antepartum cervical intraepithelial neoplasia II and III lesions. Obstet Gynecol. 1999;93:359-62.

8. Vlahos G, Rodolakis A, Diakomanolis E, Stefanidis K, Haidopoulos D, Abela K et al. Conservative Management of cervical intraepithelial neoplasia (CIN 2-3) in pregnant women. Gynecol Obstet Invest. 2002;54:78-81.

9. Paraskevaidis E, Koliopoulos G, Kalantaridou S, Pappa L, Navrozoglou I, Zikopoulos K, et al. Management and evolution of cervical intraepithelial neoplasia during pregnancy and postpartum. Eur J Obstet Gynecol and Reprod Biol. 2002;104:67-9.

10. Massad LS, Einstein MH, Huh WK, Katki HA, Kinney WK, Schiffman M, et al. 2012 updated consensus guidelines for the management of abnormal cervical cancer screening tests and cancer precursors. Obstet Gynecol. 2013;121:829-46

11. Coppolillo EF, De Ruda Vega HM, Brizuela J, Eliseht MC, Barata A, Perazzi BE. High-grade cervical neoplasia during pregnancy: diagnosis, management and postpartum findings. Acta Obstet Gynecol Scand. 2013;92:293-7.

12. Fader AN, Alward EK, Niederhauser A, Chirico C, Lesnock JL, Zwiesler DJ, et al. Cervical 7dysplasia in pregnancy: a multi-institutional evaluation. Am J Obstet Gynecol. 2010;203(2):113. e1-6.

13. Jain AG, Higgins RV, Boyle MJ. Management of low-grade squamous intraepithelial lesions during pregnancy. Am J Obstet Gynecol. 1997;177:298-302.

14. Ahdoot D, Van Nostrand KM, Nguyen NJ, Tewari DS, Kurasaki T, DiSaia PJ, et al. The effect of route of delivery on regression of abnormal cervical cytologic findings in the postpartum period. Am J Obstet Gynecol. 1998; 178:1116-20.

15. Serati M, Uccella S, Laterza RM, Salvatore S, Beretta P, Riva C, et al. Natural history of cervical intraepithelial neoplasia during pregnancy. Acta Obstet Gynecol. 2008;87:1296-300.

16. Palle C, Bangsbøll S, Andreasson B. Cervical intraepithelial neoplasia in pregnancy. Acta Obstet Gynecol Sand. 2000;79:306-10.

17. Kärrberg C, Brännström M, Strander B, Ladfors L, Rådberg T. Colposcopically directed cervical biopsy during pregnancy; minor surgical and obstetrical complications and high rates of persitence and regression. Acta Obstet Gynecol Scand. 2013;92:692-9.

18. Wright Jr TC, Massad LS, Dunton CJ, Spitzer M, Wilkinson EJ, Solomon D. 2006 consensus guidelines for the management of women with abnormal cervical cancer screening tests. Am J Obstet Gynecol. 2007;197:346-55.

19. Lurain JR, Gallup DG. Management of abnormal PAP smears in pregnancy. Obstet Gynecol. 1979;53:484-8.

20. Xavier-Júnior JC, Dufloth RM, do Vale DB, Tavares TA, Zeferino LC. Highgrade squamous intraepithelial lesions in pregnant and non-pregnant women. Eur J Obstet Gynecol Reprod Biol. 2014;175:103-6.

21. Stonehocker J. Cervical cancer screening in pregnancy. Obstet Gynecol Clin North Am. 2013:40:269-82.

22. Morice P, Uzan C, Gouy S, Verschraegen C, Haie-Meder C. Gynaecological cancers in pregnancy. Lancet. 2012;379:558-69.

23. Economos K, Perez Veridiano N, Delke I, Collado MI, Tancer MI. Abnormal cervical cytology in pregnancy: A 17-year experience. Obstet Gynecol. 1993;81:915-8.

24. Benedet JL, Selke RA, Nickerson KG. Colposcopic evaluation of abnormal Papanicolaou smears in pregnancy. Am J Obstet Gynecol. 1987;157:932-7.

25. Averette HE, Nasser N, Yankow SL, Little WA. Cervical conzation in pregnancy. Anlysis of 180 operations. Am J Obstet Gynecol. 1970;106:543-9.

26. Hannigan EV, Whitehouse 3rd HH, Atkinson WD, Becker SN. Cone biopsy during pregnancy. Obstet Gynecol. 1982;60:450-5.

27. Holowaty P, Miller AB, Rohan T, To T. Natural history of dysplasia of the uterine cervix. J Natl Cancer Inst. 1999:91:252-8.

28. Siristatidis C, Vitoratos N, Michailidis E, Syciotis C, Panagiotopoulos N, Kassanos D, et al. The role of the mode of delivery in the alteration of intrapartum pathological cervical cytologic findings during the postaprtum period. Eur J Gynecol Oncol. 2002;23:358-60.

29. Strinic T, Bukovic D, Karelovic D, Bojic L, Stipic I. The effect of delivery on regression of abnormal cervical cytologic findings. Coll Antropol. 2002;26:577-82.

30. Coppola A, Sorosky J, Casper R, Anderson B, Buller RE. The clinical course of cervical carcinoma in situ diagnosed during pregnancy. Gynecol Oncol. 1997;67:162-5

31. Ackermann S, Gehrsitz C, Melhorn G, Beckman MW. Management and course of histologically verified cervical carcinoma in situ during pregnancy. Acta Obstet Gynecol Scand. 2006:85:1134-7.

32. Everson JA, Stika CS, Lurain JR. Postpartum evolution of cervical squamous intraepithelial lesions with respect to the route of delivery. J Lower Genit Tract Dis. 2002;6:212-7.

33. Kaplan KJ, Dainty LA, Dolinsky B, Rose GS, Carlson J, McHale M, et al. Prognosis and recurrence risk for patients with cervical squamous intraepithelial lesions diagnosed during pregnancy. Cancer. 2004;102:228-32.

34. Kjellberg L, Hallmans G, Ahren AM, Johansson R, Bergman F, Wadell G, et al. Smoking, diet, pregnancy and oral contraceptive use as risk factor for cervical intraepithelial neoplasia in relation to human papillomavirus infection. Br J Cancer. 2000:82:1332-8.

35. Xavier-Júnior JC, Vale DB, Vieira LF, Lima MT, Zeferino LC, Dufloth RM. Results of screening for cervical cancer among pregnant and non-pregnant women in Brazil. Int J Gynaecol Obstet. 2015;130:36-9. 